\title{
Nontrivial Solutions for Periodic Schrödinger Equations with Sign-Changing Nonlinearities
}

\author{
Shaowei Chen, ${ }^{1}$ Lishan Lin, ${ }^{2}$ and Liqin Xiaoo \\ ${ }^{1}$ School of Mathematical Sciences, Huaqiao University, Quanzhou 362021, China \\ ${ }^{2}$ School of Applied Mathematical Sciences, Xiamen University of Technology, Xiamen 361024, China \\ Correspondence should be addressed to Shaowei Chen; swchen6@163.com
}

Received 25 September 2014; Accepted 12 December 2014

Academic Editor: Mark A. McKibben

Copyright (c) 2015 Shaowei Chen et al. This is an open access article distributed under the Creative Commons Attribution License, which permits unrestricted use, distribution, and reproduction in any medium, provided the original work is properly cited.

We prove a new infinite-dimensional linking theorem. Using this theorem, we obtain nontrivial solutions for strongly indefinite periodic Schrödinger equations with sign-changing nonlinearities.

\section{Introduction and Statement of Results}

In this paper, we consider the following semilinear Schrödinger equation:

$$
-\Delta u+V(x) u+f(x, u)=0, \quad u \in H^{1}\left(\mathbb{R}^{N}\right),
$$

where $N \geq 1$. The potential $V \in L^{\infty}\left(\mathbb{R}^{N}\right)$ is 1-periodic in $x_{j}$ for $j=1, \ldots, N$. In this case, the spectrum of the operator $-\Delta+V$ is a purely continuous spectrum bounded from below and consists of closed disjoint intervals ([1, Theorem XIII.100]). Thus, the complement $\mathbb{R} \backslash \sigma(L)$ consists of open intervals called spectral gaps. More precisely, for $V$, we assume the following.

(v) $V \in L^{\infty}\left(\mathbb{R}^{N}\right)$ is 1-periodic in $x_{j}$ for $j=1, \ldots, N, 0$ is in a spectral gap $\left(-\mu_{-1}, \mu_{1}\right)$ of $-\Delta+V$, and $-\mu_{-1}$ and $\mu_{1}$ lie in the essential spectrum of $-\Delta+V$. Denote

$$
\mu_{0}:=\min \left\{\mu_{-1}, \mu_{1}\right\} .
$$

For $f$, we assume the following.

$\left(\mathbf{f}_{\mathbf{1}}\right) f$ is a Caratheodory function in $\mathbb{R}^{N} \times \mathbb{R}$ and it is 1periodic in $x_{j}$ for $j=1, \ldots, N$. In addition, there exist constants $C>0$ and $2<p<2^{*}$ such that

$$
|f(x, t)| \leq C\left(1+|t|^{p-1}\right), \quad \forall(x, t) \in \mathbb{R}^{N} \times \mathbb{R},
$$

where

$$
2^{*}:= \begin{cases}\frac{2 N}{N-2}, & N \geq 3 \\ \infty, & N=1,2 .\end{cases}
$$

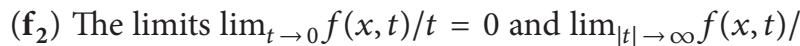
$t=+\infty$ hold uniformly for $x \in \mathbb{R}^{N}$.

Let $F(x, t)=\int_{0}^{t} f(x, s) d s, V_{-}(x)=\max \{-V(x), 0\}, x \in$ $\mathbb{R}^{N}$, and

$$
\widetilde{F}(x, t):=\frac{1}{2} t f(x, t)-F(x, t) .
$$

$\left(\mathbf{f}_{3}\right)$ There exist $\rho>0$ and $M>\rho$ such that

$$
\begin{aligned}
& \inf _{|t| \geq M / 2, x \in \mathbb{R}^{N}}\left(\frac{f(x, t)}{t}-V_{-}(x)\right)>0, \\
& \inf _{|t| \geq \rho, x \in \mathbb{R}^{N}} F(x, t) \geq 0, \\
& \inf _{\rho \leq|t| \leq M, x \in \mathbb{R}^{N}} \widetilde{F}(x, t)>0,
\end{aligned}
$$




$$
\begin{aligned}
& \sup _{|t| \leq \rho, x \in \mathbb{R}^{N}}\left|\frac{f(x, t)}{t}\right|+\left(\sup _{\rho \leq|t| \leq M, x \in \mathbb{R}^{N}} \frac{f^{2}(x, t)}{|\widetilde{F}(x, t)|}\right)^{1 / 2} \\
& \cdot\left(\sup _{|t| \leq \rho, x \in \mathbb{R}^{N}} \frac{|\widetilde{F}(x, t)|}{t^{2}}\right)^{1 / 2}<\mu_{0} .
\end{aligned}
$$

A solution $u$ of (1) is called nontrivial if $u \neq \equiv$. Our main result is the following theorem.

Theorem 1. Suppose that $(v)$ and $\left(f_{1}\right)-\left(f_{3}\right)$ are satisfied. Then problem (1) has a nontrivial solution.

As an application of this theorem, we have the following corollary.

Corollary 2. Suppose that $2<p<q<2^{*}$ and (v) is satisfied. Then, there exists $\lambda_{0}>0$ such that, for $0<\lambda<\lambda_{0}$,

$$
-\Delta u+V u=\lambda|u|^{p-2} u-|u|^{q-2} u
$$

has a nontrivial solution.

Remark 3. This corollary shows that, under assumptions $\left(\mathbf{f}_{1}\right)-\left(\mathbf{f}_{\mathbf{3}}\right)$, the nonlinearity $f$ may be sign-changing.

Under assumption $(\mathbf{v})$, the quadratic form $\int_{\mathbb{R}^{N}}\left(|\nabla u|^{2}+\right.$ $\left.V(x) u^{2}\right) d x$ has infinite-dimensional negative and positive spaces. This case is called strongly indefinite. Semilinear periodic Schrödinger equations with strongly indefinite linear parts have attracted considerable attention in recent years due to their numerous applications in mathematical physics. In [2], the authors used a dual variational method to obtain a nontrivial solution of (1) with $f(x, t)=-W(x)|t|^{p-2} t$, where $W$ is a perturbed periodic function and $2<p<2^{*}$. In [3], Troestler and Willem used critical point theory to obtain a nontrivial solution of (1) by assuming that $f \in C^{1}\left(\mathbb{R}^{N} \times \mathbb{R}\right)$, $\left|\partial_{t} f(x, t)\right| \leq C\left(|t|^{p-2}+|t|^{q-2}\right)$ for some $2<p<q<2^{*}$ and $g=-f$ satisfy the so-called Ambrosetti-Rabinowitz condition $0<\gamma G(x, t) \leq \operatorname{tg}(x, t), \forall(x, t) \in \mathbb{R}^{N} \times(\mathbb{R} \backslash$ $\{0\})$, where $G(x, t)=\int_{0}^{t} g(x, s) d s$. Kryszewski and Szulkin [4] subsequently proved a new infinite-dimensional linking theorem. Using this, they generalized Troestler and Willem's result by assuming that $f \in C\left(\mathbb{R}^{N} \times \mathbb{R}\right)$ and $g=-f$ satisfy the Ambrosetti-Rabinowitz condition. Similar results were obtained by Pankov and Pflüger in $[5,6]$ by using an approximation method and a variant of the Nehari method. Equation (1), with asymptotically linear nonlinearities and other superlinear nonlinearities, has also been studied by several researchers. The interested readers can see [7-10] for the asymptotically linear case and [9, 11-17] for the superlinear case. Moreover, (1), with 0 belonging to the spectrum of $-\Delta+V$, was investigated in $[8,18,19]$. It is worth mentioning that methods of studying strongly indefinite periodic Schrödinger equations can shed light on other strongly indefinite problems, such as Hamiltonian systems and discrete nonlinear Schrödinger equations. The reader can consult $[8,20]$ or $[21]$ for more details.

All existence results for (1) mentioned above are obtained under the assumption that $t f$ does not change sign in $\mathbb{R}^{N} \times$ $\mathbb{R}$; that is, $t f(x, t) \geq 0$ in $\mathbb{R}^{N} \times \mathbb{R}$ or $t f(x, t) \leq 0$ in $\mathbb{R}^{N} \times$ $\mathbb{R}$. However, under our assumptions $\left(\mathbf{f}_{1}\right)-\left(\mathbf{f}_{\mathbf{3}}\right), t f(x, t)$ can be negative in $\{(x, t)|| t \mid<\rho\}$. Together with (7), this implies that $t f(x, \cdot)$ may change sign in $\mathbb{R}$. As we know, this situation has never before been studied. And it is the novelty of our main results of Theorem 1 and Corollary 2. Theorem 1 can be seen as a generalization of Theorem 3 of [22].

The difficulties of (1) with regard to sign-changing nonlinearity are due to two aspects. The first is that the classical infinite-dimensional linking theorem (see [23, Theorem 6.10] or [4]) cannot be used to deal with (1) in this case. To use this linking theorem, the functional corresponding to (1) must satisfy a certain upper semicontinuous assumption. However, when $f(x, t)$ is sign-changing, the functional corresponding to (1) does not satisfy such a assumption. The second aspect that causes problems is that sign-changing nonlinearity creates more difficulties in proving the boundedness of the Palais-Smale sequence.

In this paper, we provide a variant of the classical infinitedimensional linking theorem (see Theorem 7). This theorem and its corollary replace the upper semicontinuous assumption in the classical infinite-dimensional linking theorem ([23, Theorem 6.10]) with other assumptions. We present the theorem and its proof in Section 2. Using this theorem, $a(\bar{C})_{c}$ sequence (see Definition 6) of (1) is obtained. Under $\left(\mathbf{f}_{\mathbf{1}}\right)-\left(\mathbf{f}_{\mathbf{3}}\right)$, we can prove that this sequence is bounded in $H^{1}\left(\mathbb{R}^{N}\right)$ (see Lemma 12). We then obtain a nontrivial solution of (1) from the $(\bar{C})_{c}$ sequence by using the concentration-compactness principle.

Notation. $B_{r}(a)$ denotes an open ball of radius $r$ and center $a$. For a Banach space $E$, we denote the dual space of $E$ by $E^{\prime}$ and denote strong and weak convergence in $E$ by $\rightarrow$ and $\rightarrow$, respectively. For $\varphi \in C^{1}(E ; \mathbb{R})$, we denote the Fréchet derivative of $\varphi$ at $u$ by $\varphi^{\prime}(u)$. The Gateaux derivative of $\varphi$ is denoted by $\left\langle\varphi^{\prime}(u), v\right\rangle, \forall u, v \in E . L^{p}\left(\mathbb{R}^{N}\right)$ denotes the standard $L^{p}$ space $(1 \leq p \leq \infty)$, and $H^{1}\left(\mathbb{R}^{N}\right)$ denotes the standard Sobolev space with norm $\|u\|_{H^{1}}=\left(\int_{\mathbb{R}^{N}}\left(|\nabla u|^{2}+\right.\right.$ $\left.\left.u^{2}\right) d x\right)^{1 / 2}$. We use $O(h), o(h)$ to mean $|O(h)| \leq C|h|$ and $o(h) /|h| \rightarrow 0$.

\section{A New Infinite-Dimensional Linking Theorem}

In this section, we give a variant linking theorem which is a generalization of the classical infinite-dimensional linking theorem of [23, Theorem 6.10] (see also [4]).

Before stating this theorem, we give some notations and definitions.

Let $X$ be a separable Hilbert space with inner product $(\cdot, \cdot)$ and norm $\|\cdot\|$, respectively. $X^{ \pm}$are closed subspaces of $X$ and 
$X=X^{+} \oplus X^{-}$. Let $\left\{e_{k}^{-}\right\}$be the total orthonormal sequence in $X^{-}$. Let

$$
\begin{aligned}
& Q: X \longrightarrow X^{+}, \\
& P: X \longrightarrow X^{-}
\end{aligned}
$$

be the orthogonal projections. We define

$$
\|\mid u\| \|=\max \left\{\|Q u\|, \sum_{k=1}^{\infty} \frac{1}{2^{k+1}}\left|\left(P u, e_{k}^{-}\right)\right|\right\}
$$

on $X$. Then

$$
\|Q u\| \leq\|u\|\|\leq\| u \|, \quad \forall u \in X,
$$

and if $\left\|u_{n}\right\|$ is bounded and $\left\|\mid u_{n}-u\right\| \| \rightarrow 0$, then $\left\{u_{n}\right\}$ weakly converges to $u$ in $X$. The topology generated by $\||\cdot|||$ is denoted by $\tau$, and all topological notations related to it will include this symbol.

Let $R>r>0$ and $z_{0} \in X^{+}$with $\left\|z_{0}\right\|=1$. Set

$$
\begin{aligned}
& N=\left\{z \in X^{+} \mid\|z\|=r\right\}, \\
& M=\left\{z+t z_{0} \mid z \in X^{-}, t \geq 0,\left\|z+t z_{0}\right\| \leq R\right\} .
\end{aligned}
$$

Then, $M$ is a submanifold of $X^{-} \oplus \mathbb{R}^{+} u_{0}$ with boundary

$$
\begin{aligned}
\partial M= & \left\{z \in X^{-} \mid\|z\| \leq R\right\} \\
& \cup\left\{z+t z_{0} \mid z \in X^{-}, t>0,\left\|z+t z_{0}\right\|=R\right\} .
\end{aligned}
$$

Definition 4. A functional $\phi \in C^{1}(X, \mathbb{R})$ is called $\tau$-upper semicontinuous if, for any $a \in \mathbb{R}, \phi_{a}:=\{u \in X \mid \phi(u) \geq a\}$ is a $\tau$-closed set. And $\phi^{\prime}$ is called weakly sequentially continuous if $u \in X$ and $\left\{u_{n}\right\} \subset X$ are such that $u_{n} \rightarrow u$; then, for any $\varphi \in X,\left\langle\phi^{\prime}\left(u_{n}\right), \varphi\right\rangle \rightarrow\left\langle\phi^{\prime}(u), \varphi\right\rangle$.

Remark 5. If a functional $\phi \in C^{1}(X, \mathbb{R})$ has the form

$$
\phi(u)=c_{1}\|Q u\|^{2}-c_{2}\|P u\|^{2}-\psi(u)
$$

with $c_{1}>0, c_{2}>0$ and $\psi \in C^{1}(X, \mathbb{R})$ is weakly sequentially lower semicontinuous, that is, if $u_{n} \rightarrow u$, then $\liminf _{n \rightarrow \infty} \psi\left(u_{n}\right) \geq \psi(u)$, then by Remark 2.1 (iii) of [4], $\phi$ is $\tau$-upper semicontinuous.

Definition 6. Let $\phi \in C^{1}(X, \mathbb{R})$. A sequence $\left\{u_{n}\right\} \subset X$ is called a $(\bar{C})_{c}$ sequence for $\phi$, if

$$
\begin{aligned}
\limsup _{n \rightarrow \infty} \phi\left(u_{n}\right) \leq c, & \\
\left(1+\left\|u_{n}\right\|\right)\left\|\phi^{\prime}\left(u_{n}\right)\right\|_{X^{\prime}} & \longrightarrow 0, \\
\text { as } n & \longrightarrow \infty .
\end{aligned}
$$

The main results of this section are the following theorem and corollary.
Theorem 7. If $H \in C^{1}(X, \mathbb{R})$ satisfies the following

(a) $H^{\prime}$ is weakly sequentially continuous,

(b) there exists a $\tau$-upper semicontinuous functional J such that $H(u) \leq J(u), \forall u \in X$,

(c) there exist $u_{0} \in X^{+}$with $\left\|u_{0}\right\|=1$ and $R>r>0$ such that

$$
\begin{aligned}
& \inf _{N} H>\sup _{\partial M} H, \\
& \sup _{M} H<+\infty,
\end{aligned}
$$

then, for any $\kappa<\inf _{N} H$, there exists a $(\bar{C})_{c}$ sequence $\left\{u_{n}\right\}$ of $H$ with $c=\sup _{M} H$ such that

$$
\inf _{n} J\left(u_{n}\right) \geq \kappa .
$$

Corollary 8. Let $H$ and J satisfy assumptions (a), (b), and (c) in Theorem 7. If

$$
J(0)<\inf _{N} H,
$$

then there exist $\delta>0$ and $a(\bar{C})_{c}$ sequence $\left\{u_{n}\right\}$ of $H$ with $c=$ $\sup _{M} H$ such that

$$
\inf _{n}||\left|u_{n}\right| \| \geq \delta .
$$

Remark 9. (i) To use the classical infinite-dimensional linking theorems, such as Theorem 3.4 of [4] or Theorem 6.10 of [23], to get a Palais-Smale sequence or $(\bar{C})_{c}$ sequence for a functional satisfying the linking condition (18), this functional should be $\tau$-upper semicontinuous. This assumption precludes applying these classical infinite-dimensional linking theorems to problems possessing wider class of nonlinearities, such as nonlinear Schrödinger equations with sign-changing nonlinearities.

(ii) $(\bar{C})_{c}$ sequence (see Definition 6) can be seen as a weighted variation of Palais-Smale sequence. It plays important role in proving the boundedness. For example, the $(\bar{C})_{c}$ sequence in Lemma 11 of this paper cannot be replaced with Palais-Smale sequence.

(iii) This theorem and its corollary are new infinitedimensional linking theorems different from the one published in [24, Theorem 1.3].

Proof of Theorem 7. Arguing indirectly, assume that the result does not hold. Then, there exist $\epsilon>0$ and $\kappa_{0}<\inf _{N} H$ such that

$$
(1+\|u\|)\left\|H^{\prime}(u)\right\|_{X^{\prime}} \geq \epsilon, \quad \forall u \in E,
$$

where

$$
\begin{aligned}
& E=\{u \in X \mid H(u) \leq d+\epsilon\} \cap\left\{u \in X \mid J(u) \geq \kappa_{0}\right\}, \\
& d=\sup _{M} H .
\end{aligned}
$$


Since $H \leq J$ in $X$, we deduce that

$$
\begin{aligned}
H_{\kappa_{0}}:= & \left\{u \in X \mid H(u) \geq \kappa_{0}\right\} \\
& \subset J_{\kappa_{0}}:=\left\{u \in X \mid J(u) \geq \kappa_{0}\right\} .
\end{aligned}
$$

Therefore,

$$
H_{\kappa_{0}}^{d+\epsilon}:=\left\{u \in X \mid \kappa_{0} \leq H(u) \leq d+\epsilon\right\} \subset E .
$$

Step 1. A vector field in a $\tau$-neighborhood of

$$
H^{d+\epsilon}:=\{u \in X \mid H(u) \leq d+\epsilon\} .
$$

Let

$$
\begin{aligned}
b & =\inf _{N} H, \\
T & =\frac{2\left(d+\epsilon-\kappa_{0}\right)}{\epsilon}, \\
R^{\prime} & =\left(1+\sup _{u \in M}\|u\|\right) e^{2 T}, \\
B_{R^{\prime}} & =\left\{u \in X \mid\|u\| \leq R^{\prime}\right\} .
\end{aligned}
$$

For every $u \in E \cap B_{R^{\prime}}$, there exists $\phi_{u} \in X$ with $\left\|\phi_{u}\right\|=1$ such that $\left\langle H^{\prime}(u), \phi_{u}\right\rangle \geq(3 / 4)\left\|H^{\prime}(u)\right\|_{X^{\prime}}$. Then, (23) implies that

$$
(1+\|u\|)\left\langle H^{\prime}(u), \phi_{u}\right\rangle>\frac{1}{2} \epsilon .
$$

From the definition of \|\|$\cdot \|$, we deduce that if a sequence $\left\{u_{n}\right\} \subset E \cap B_{R^{\prime}} \tau$-converges to $u \in X$, that is, $\| u_{n}-$ $u \| l \mid 0$, then $u_{n} \rightarrow u$ in $X$ (see Remark 6.1 of [23]). By the weakly sequential continuity of $H^{\prime}$, we get that for any $\varphi \in X,\left\langle H^{\prime}\left(u_{n}\right), \varphi\right\rangle \rightarrow\left\langle H^{\prime}(u), \varphi\right\rangle$. This implies that $H^{\prime}$ is $\tau$-sequentially continuous in $E \cap B_{R^{\prime}}$. By (30), the $\tau$-sequential continuity of $H^{\prime}$ in $E \cap B_{R^{\prime}}$, and the weakly lower semicontinuity of the norm $\|\cdot\|$, we get that there exists a $\tau$-open neighborhood $V_{u}$ of $u$ such that

$$
\begin{aligned}
& \left\langle H^{\prime}(v),(1+\|u\|) \phi_{u}\right\rangle>\frac{1}{2} \epsilon, \quad \forall v \in V_{u} \cap\left(E \cap B_{R^{\prime}}\right), \\
& \left\|(1+\|u\|) \phi_{u}\right\|=1+\|u\| \leq 2(1+\|v\|), \quad \forall v \in V_{u} .
\end{aligned}
$$

Because $B_{R^{\prime}}$ is a bounded convex closed set in the Hilbert space $X, B_{R^{\prime}}$ is a $\tau$-closed set. It follows that $X \backslash B_{R^{\prime}}$ is a $\tau$-open set. Moreover, since $J$ is a $\tau$-upper semicontinuous functional, $\left\{u \in X \mid J(u) \geq \kappa_{0}\right\}$ is a $\tau$-closed set. It follows that

$$
U=\left\{u \in X \mid J(u)<\kappa_{0}\right\}
$$

is a $\tau$-open set. Therefore, the family

$$
\mathcal{N}=\left\{V_{u} \mid u \in E \cap B_{R^{\prime}}\right\} \cup\left\{X \backslash B_{R^{\prime}}\right\} \cup\{U\}
$$

is a $\tau$-open covering of $H^{d+\epsilon}$. Let

$$
\mathscr{V}=\left(\bigcup_{u \in E \cap B_{R^{\prime}}} V_{u}\right) \cup\left(X \backslash B_{R^{\prime}}\right) \cup U .
$$

Then, $\mathscr{V}$ is a $\tau$-open neighborhood of $H^{d+\epsilon}$.
Since $\mathscr{V}$ is metric, hence paracompact, there exists a local finite $\tau$-open covering $\mathscr{M}=\left\{M_{i} \mid i \in \Lambda\right\}$ of $\mathscr{V}$ finer than $\mathcal{N}$. If $M_{i} \subset V_{u_{i}}$ for some $u_{i} \in E \cap B_{R^{\prime}}$, we choose $\omega_{i}=\left(1+\left\|u_{i}\right\|\right) \phi_{u_{i}}$ and if $M_{i} \subset X \backslash B_{R^{\prime}}$ or if $M_{i} \subset U$, we choose $\omega_{i}=0$. Let $\left\{\lambda_{i} \mid i \in I\right\}$ be a $\tau$-Lipschitz continuous partition of unity subordinated to $\mathscr{M}$. And let

$$
\xi(u):=\sum_{i \in I} \lambda_{i}(u) \omega_{i}, \quad u \in \mathscr{V} .
$$

Since the $\tau$-open covering $\mathscr{M}$ of $\mathscr{V}$ is local finite, each $u \in \mathscr{V}$ belongs to only finitely many sets $M_{i}$. Therefore, for every $u \in$ $\mathscr{V}$, the sum in (35) is a finite sum. This implies the following.

(a) For any $u \in \mathscr{V}$, there exist a $\tau$-open neighborhood $U_{u} \subset \mathscr{V}$ of $u$ and $L_{u}>0$ such that $\xi\left(U_{u}\right)$ is contained in a finite-dimensional subspace of $X$ and

$\|\xi(v)-\xi(w)\| \leq L_{u}\|v-w\|, \quad \forall v, w \in U_{u}$.

This means that $\xi$ is locally Lipschitz continuous and $\tau$-locally Lipschitz continuous.

Moreover, by the definition of $\xi$ and (31), we get the following.

(b) For every $u \in \mathscr{V}$,

$$
\begin{aligned}
\|\xi(u)\| & \leq 2(1+\|u\|), \\
\left\langle H^{\prime}(u), \xi(u)\right\rangle & \geq 0
\end{aligned}
$$

and for every $u \in E \cap B_{R^{\prime}}$,

$$
\left\langle H^{\prime}(u), \xi(u)\right\rangle>\frac{1}{2} \epsilon .
$$

Step 2. From (36) and the fact that \|\|$v\|\| \leq\|v\|, \forall v \in X$, we have

$$
\|\xi(v)-\xi(w)\| \leq L_{u}\|v-w\|, \quad \forall v, w \in U_{u} .
$$

This implies that $\xi$ is a local Lipschitz mapping under the $\|\cdot\|$ norm. Then, by the standard theory of ordinary differential equation in Banach space, we deduce that the following initial value problem

$$
\begin{aligned}
\frac{d \eta}{d t} & =-\xi(\eta), \\
\eta(0, u) & =u \in \mathscr{V},
\end{aligned}
$$

has a unique solution in $\mathscr{V}$, denoted by $\eta(t, u)$, with the right maximal interval of existence $[0, T(u))$. Furthermore, using (36) and the Gronwall inequality (see, e.g., Lemma 6.9 of [23]), the similar argument as the proof of (c) in [23, Lemma 6.8 ] yields that

(A) $\eta$ is $\tau$-continuous; that is, if $u_{n} \in \mathscr{V}, u_{0} \in \mathscr{V}, 0 \leq t_{n}<$ $T\left(u_{n}\right)$, and $0 \leq t_{0}<T\left(u_{0}\right)$ satisfy $\left\|\mid u_{n}-u_{0}\right\| \rightarrow 0$ and $t_{n} \rightarrow t_{0}$, then $\left\|\left|\eta\left(t_{n}, u_{n}\right)-\eta\left(t_{0}, u_{0}\right)\right|\right\| \rightarrow 0$. 
From $\left\langle H^{\prime}(u), \xi(u)\right\rangle \geq 0, \forall u \in \mathscr{V}$ (see (37)), we have

$$
\frac{d}{d t} H(\eta(t, u))=\left\langle H^{\prime}(\eta(t, u)), \eta^{\prime}(t, u)\right\rangle \leq 0 .
$$

Therefore, $H$ is nonincreasing along the flow $\eta$. It follows that $\{\eta(t, u) \mid 0 \leq t \leq T(u)\} \subset H^{d+\epsilon}$ if $u \in H^{d+\epsilon}$; that is, for any $u \in H^{d+\epsilon}$ and any $t \geq 0, \eta(t, u)$ is still in $H^{d+\epsilon}$. Moreover, since $\mathscr{V}$ is a neighborhood of $H^{d+\epsilon}, \eta(t, u) \in H^{d+\epsilon} \subset \mathscr{V}$ for any $u \in H^{d+\epsilon}$ and $t \geq 0$; that is, the flow $\eta(t, u)$ with $u \in H^{d+\epsilon}$ cannot leave $\mathscr{V}$. Therefore, $H^{d+\epsilon}$ is an invariant set of the flow $\eta$. Then, $\|\xi(u)\| \leq 2(1+\|u\|), \forall u \in \mathscr{V}$ (see (37)) and Theorem 5.6.1 of [25] implies that, for any $u \in H^{d+\epsilon}, T(u)=+\infty$.

Step 3. We will prove that

$$
\{\eta(t, u) \mid 0 \leq t \leq T, u \in M\} \subset B_{R^{\prime}},
$$

where $T$ is defined in (28).

Let $u \in H^{d+\epsilon}$. By the result in Step 2, we have $T(u)=+\infty$ and

$$
\eta(t, u)=u-\int_{0}^{t} \xi(\eta(s, u)) d s, \quad \forall t \in[0,+\infty) .
$$

Together with $\|\xi(u)\| \leq 2(1+\|u\|), \forall u \in \mathscr{V}$ (see (37)), this yields

$$
\begin{aligned}
\|\eta(t, u)\| & \leq\|u\|+\int_{0}^{t}\|\xi(\eta(s, u))\| d s \\
& \leq\|u\|+2 \int_{0}^{t}(1+\|\eta(s, u)\|) d s .
\end{aligned}
$$

Then, by the Gronwall inequality (see, e.g., Lemma 6.9 of [23]), we get that

$$
\|\eta(t, u)\| \leq(1+\|u\|) e^{2 t}-1, \quad \forall t \in[0,+\infty) .
$$

Since $M \subset H^{d+\epsilon}$, by (45) and the definition of $R^{\prime}$ (see (29)), we obtain (42).

Step 4. From (26) and (38), we deduce that

$$
\left\langle H^{\prime}(u),-\xi(u)\right\rangle<-\frac{1}{2} \epsilon, \quad \forall u \in H_{\kappa_{0}}^{d+\epsilon} \cap B_{R^{\prime}} .
$$

We show that, for any $u \in M, H(\eta(T, u)) \leq \kappa_{0}$. Arguing indirectly, assume that this was not true. Then, there exists $u \in M$ such that $H(\eta(T, u))>\kappa_{0}$. Since $H$ is nonincreasing along the flow $\eta$, from (42), we deduce that $\{\eta(t, u) \mid 0 \leq t \leq$ $T\} \subset H_{\kappa_{0}}^{d+\epsilon} \cap B_{R^{\prime}}$. Then, by (46),

$$
\begin{aligned}
H(\eta(T, u))= & H(\eta(0, u)) \\
& +\int_{0}^{T}\left\langle H^{\prime}(\eta(s, u)),-\xi(\eta(s, u))\right\rangle d s \\
\leq & H(\eta(0, u))+\int_{0}^{T}\left(-\frac{1}{2} \epsilon\right) d s \\
\leq & d+\epsilon-\frac{1}{2} \epsilon T=\kappa_{0} .
\end{aligned}
$$

This contradicts $H(\eta(T, u))>\kappa_{0}$. Therefore, we have

(B) $\eta(T, M) \subset H^{\kappa_{0}}:=\left\{u \in X \mid H(u) \leq \kappa_{0}\right\}$.
Moreover, using the result (a) in Step 1 and the fact that $\eta$ is $\tau$-continuous (see (A)), the similar argument as the proof of the result (b) of [23, Lemma 6.8] yields that

(C) each point $(t, u) \in[0, T] \times H^{d+\epsilon}$ has a $\tau$-neighborhood $N_{(t, u)}$ such that

$$
\left\{v-\eta(s, v) \mid(s, v) \in N_{(t, u)} \cap\left([0, T] \times H^{d+\epsilon}\right)\right\}
$$

is contained in a finite-dimensional subspace of $X$.

Step 5. Let

$$
\begin{aligned}
& h:[0, T] \times M \longrightarrow X, \\
& h(t, u)=P \eta(t, u)+(\|Q \eta(t, u)\|-r) u_{0},
\end{aligned}
$$

where $P, Q, r$, and $u_{0}$ are defined in (11), (14), and (15). Then

$$
0 \in h(t, M) \Longleftrightarrow \eta(t, M) \cap N \neq \emptyset .
$$

From $\inf _{N} H>\sup _{\partial M} H$ (see (18)) and the fact that, for any $u \in X$, the function $H(\eta(\cdot, u))$ is nonincreasing, we deduce that $\inf _{N} H>\sup _{u \in \partial M} H(\eta(t, u)), \forall t \in[0, T]$. Therefore,

$$
0 \notin h(t, \partial M), \quad \forall t \in[0, T] .
$$

Since $\eta$ has properties (A) and (C) obtained in Steps 2 and 4, respectively, and $h$ satisfies (51), there is an appropriate degree theory for $\operatorname{deg}(h(t, \cdot), M, 0)$ (see Proposition 6.4 and Theorem 6.6 of [23]). Then, the same argument as the proof of Theorem 6.10 of [23] yields that

$$
\operatorname{deg}(h(T, \cdot), M, 0)=\operatorname{deg}(h(0, \cdot), M, 0) \neq 0 .
$$

It follows that $0 \in h(T, M)$ and $\eta(T, M) \cap N \neq \emptyset$. Therefore, there exists $u \in M$ such that $H(\eta(T, u)) \geq b$. It contradicts property (B) obtained in Step 4 , since $\kappa_{0}<b$. This completes the proof of this theorem.

Proof of Corollary 8. Since $J(0)<\inf _{N} H$, we can choose $\kappa$ such that $J(0)<\kappa<\inf _{N} H$. By Theorem 7 , there exists a $(\bar{C})_{c}$ sequence $\left\{u_{n}\right\}$ of $H$ such that $c=\sup _{M} H$ and $\inf _{n} J\left(u_{n}\right) \geq \kappa$. From $J(0)<\kappa<\inf _{N} H$, we deduce that $0 \in\{u \in X \mid J(u)<$ $\kappa\}$. Since $J$ is $\tau$-upper semicontinuous, $\{u \in X \mid J(u)<\kappa\}$ is a $\tau$-open set. Therefore, there exists $\delta>0$ such that

$$
\{u \in X|\|| u||<\delta\} \subset\{u \in X \mid J(u)<\kappa\} .
$$

Since $\inf _{n} J\left(u_{n}\right) \geq \kappa$, by (53), we obtain $\inf _{n}\left|\left\|u_{n} \mid\right\| \geq \delta\right.$.

\section{Variational and Linking Structure for (1)}

Under assumptions $(\mathbf{v}),\left(\mathbf{f}_{1}\right)$ and the first part of $\left(\mathbf{f}_{2}\right)$, the functional

$$
\begin{aligned}
J(u)= & \frac{1}{2} \int_{\mathbb{R}^{N}}|\nabla u|^{2} d x+\frac{1}{2} \int_{\mathbb{R}^{N}} V(x) u^{2} d x \\
& +\int_{\mathbb{R}^{N}} F(x, u) d x
\end{aligned}
$$


belongs to class $C^{1}$ for $X:=H^{1}\left(\mathbb{R}^{N}\right)$. The derivative of $J$ is

$$
\begin{aligned}
\left\langle J^{\prime}(u), v\right\rangle= & \int_{\mathbb{R}^{N}}(\nabla u \nabla v+V(x) u v) d x \\
& +\int_{\mathbb{R}^{N}} f(x, u) v d x, \quad u, v \in H^{1}\left(\mathbb{R}^{N}\right)
\end{aligned}
$$

and the critical points of $J$ are weak solutions of (1).

There is a standard variational setting for the quadratic form $\int_{\mathbb{R}^{N}}\left(|\nabla u|^{2}+V(x) u^{2}\right) d x$. For the reader's convenience, we state it here. The interested reader should consult [9] or [8] for more details.

Assume that (v) holds and let $S=-\Delta+V$ be the selfadjoint operator acting on $L^{2}\left(\mathbb{R}^{N}\right)$ with domain $D(S)=$ $H^{2}\left(\mathbb{R}^{N}\right)$. By virtue of $(\mathbf{v})$, we have the orthogonal decomposition

$$
L^{2}=L^{2}\left(\mathbb{R}^{N}\right)=L_{1}+L_{2}
$$

such that $S$ is positive (resp., negative) in $L_{1}$ (resp., in $L_{2}$ ). Let $X=D\left(|S|^{1 / 2}\right)$ be equipped with the inner product

$$
(u, v)=\left(|S|^{1 / 2} u,|S|^{1 / 2} v\right)_{L^{2}}
$$

and norm $\|u\|=\left\||S|^{1 / 2} u\right\|_{L^{2}}$, where $(\cdot, \cdot)_{L^{2}}$ denotes the inner product of $L^{2}$. From (v),

$$
X=H^{1}\left(\mathbb{R}^{N}\right)
$$

with equivalent norms. Therefore, $X$ continuously embeds into $L^{q}\left(\mathbb{R}^{N}\right)$ for all $2 \leq q \leq 2^{*}$. In addition, we have the decomposition

$$
X=Y \oplus Z \text {, }
$$

where $Y=X \cap L_{1}$ and $Z=X \cap L_{2}$ and $Y, Z$ are orthogonal with respect to both $(\cdot, \cdot)_{L^{2}}$ and $(\cdot, \cdot)$. Let $P: X \rightarrow Y$ and $Q: X \rightarrow Z$ be orthogonal projections. Therefore, for every $u \in X$, there is a unique decomposition

$$
u=P u+Q u
$$

with $(P u, Q u)=0$ and

$$
\begin{gathered}
\int_{\mathbb{R}^{N}}|\nabla u|^{2} d x+\int_{\mathbb{R}^{N}} V(x) u^{2} d x \\
=\|P u\|^{2}-\|Q u\|^{2}, \quad u \in X .
\end{gathered}
$$

Moreover,

$$
\begin{gathered}
\mu_{1}\|P u\|_{L^{2}}^{2} \leq\|P u\|^{2}, \quad \forall u \in X, \\
\mu_{-1}\|Q u\|_{L^{2}}^{2} \leq\|Q u\|^{2}, \quad \forall u \in X .
\end{gathered}
$$

Therefore,

$$
\mu_{0}\|u\|_{L^{2}}^{2} \leq\|u\|^{2}, \quad \forall u \in X
$$

We denote

$$
\begin{aligned}
& X^{+}:=Z, \\
& X^{-}:=Y, \\
& u^{+}:=Q u, \\
& u^{-}:=P u .
\end{aligned}
$$

Let

$$
\Phi(u)=-J(u), \quad u \in X
$$

Then, by (54) and (61), $\Phi$ can be written as

$$
\begin{aligned}
\Phi(u)= & \frac{1}{2}\left(\left\|u^{+}\right\|^{2}-\left\|u^{-}\right\|^{2}\right) \\
& -\int_{\mathbb{R}^{N}} F(x, u) d x, \quad u \in X .
\end{aligned}
$$

The derivative of $\Phi$ is given by

$$
\begin{aligned}
\left\langle\Phi^{\prime}(u), v\right\rangle= & \left(u^{+}, v\right)-\left(u^{-}, v\right) \\
& -\int_{\mathbb{R}^{N}} f(x, u) v d x, \quad \forall u, v \in X .
\end{aligned}
$$

Let $\left\{e_{k}^{-}\right\}$be the total orthonormal sequence in $X^{-}$and let ||$|\cdot|||$ be the norm defined by (12). The topology generated by ||$|\cdot|||$ is denoted by $\tau$, and all topological notations related to it will include this symbol.

Lemma 10. Suppose (v) and $\left(f_{1}\right)-\left(f_{3}\right)$ are satisfied. Then

(i) there exist $\delta>0, R>r>0$, and $u_{0} \in X^{+}$with $\left\|u_{0}\right\|=1$ such that

$$
\inf _{N} \Phi>\sup _{\partial M} \Phi
$$

where $N, M$, and $\partial M$ are defined in (14) and (15);

(ii) there exists a $\tau$-upper sem-continuous functional (see Definition 4) $J$ defined in $X$ such that $J(0)<\inf _{N} \Phi$ and $\Phi \leq J$ in $X$.

Proof. We divide the proof into several steps.

Step 1. We will prove that there exists $r>0$ such that $\inf _{N} \Phi>$ 0 .

From $\left(\mathbf{f}_{1}\right)$ and $\left(\mathbf{f}_{2}\right)$, we deduce that, for any $\epsilon>0$, there exists $C_{\epsilon}>0$ such that

$$
|F(x, t)| \leq \epsilon t^{2}+C_{\epsilon}|t|^{p}, \quad \forall t \in \mathbb{R} .
$$

Then by the Sobolev inequality $\|u\|_{L^{p}\left(\mathbb{R}^{N}\right)} \leq C\|u\|, \forall u \in X$, and the definition of $\Phi$ (see (66)), there exists $C^{\prime}>0$ such that, for any $u \in X^{+}$,

$$
\begin{aligned}
\Phi(u) & \geq \frac{1}{2}\|u\|^{2}-C^{\prime} \epsilon\|u\|^{2}-C^{\prime} C_{\epsilon}\|u\|^{p} \\
& =\left(\frac{1}{2}-C^{\prime} \varepsilon\right)\|u\|^{2}-C^{\prime} C_{\epsilon}\|u\|^{p}
\end{aligned}
$$


Choose $\epsilon=1 / 4 C^{\prime}$ in (70) and let $r=\left(8 C^{\prime} C_{\epsilon}\right)^{-1 /(p-2)}$. We then see that, for $N=\left\{u \in X^{+} \mid\|u\|=r\right\}$,

$$
\inf _{N} \Phi \frac{r^{2}}{8}>0 .
$$

Step 2. We will prove that

$$
\Phi(u) \leq 0, \quad \forall u \in X^{-} .
$$

Let

$$
F_{1}(x, t)= \begin{cases}F(x, t), & |t| \leq \rho, x \in \mathbb{R}^{N} \\ 0, & |t|>\rho, x \in \mathbb{R}^{N}\end{cases}
$$

and $F_{2}=F-F_{1}$. From (7), we deduce $F_{2} \geq 0$ in $\mathbb{R}^{N} \times \mathbb{R}$. Let

$$
A:=\sup _{|t| \leq \rho, x \in \mathbb{R}^{N}}\left|\frac{f(x, t)}{t}\right| .
$$

From (9), we have

$$
A<\mu_{0} .
$$

Then,

$$
\left|F_{1}(x, t)\right| \leq \frac{A}{2} t^{2}, \quad \forall(x, t) \in \mathbb{R}^{N} \times \mathbb{R} .
$$

From (76), (63), (66), and the fact that $F_{2} \geq 0$ in $\mathbb{R}^{N} \times \mathbb{R}$, we deduce that, for any $u \in X^{-}$,

$$
\begin{aligned}
\Phi(u) & =-\frac{1}{2}\|u\|^{2}-\int_{\mathbb{R}^{N}} F_{1}(x, u) d x-\int_{\mathbb{R}^{N}} F_{2}(x, u) d x \\
& \leq-\frac{1}{2}\|u\|^{2}+\frac{A}{2}\|u\|_{L^{2}}^{2} \leq-\frac{1}{2}\left(1-\frac{A}{\mu_{0}}\right)\|u\|^{2} \leq 0 .
\end{aligned}
$$

Now, we prove that $\Phi(u) \rightarrow-\infty$ as $\|u\| \rightarrow \infty$ and $u \epsilon$ $X^{-} \oplus \mathbb{R}^{+} u_{0}$. Together with (72), this yields that there exists $R>r$ such that $\sup _{\partial M} \Phi \leq 0<\inf _{N} \Phi$.

Arguing indirectly, assume that, for some sequence $u_{n} \in$ $X^{-} \oplus \mathbb{R}^{+} u_{0}$ with $\left\|u_{n}\right\| \rightarrow+\infty$, there is $\mathscr{L}>0$ such that $\Phi\left(u_{n}\right) \geq-\mathscr{L}$ for all $n$. Then, setting $w_{n}=u_{n} /\left\|u_{n}\right\|$, we have $\left\|w_{n}\right\|=1$ and, up to a subsequence, $w_{n} \rightarrow w, w_{n}^{-} \rightarrow w^{-} \in X^{-}$ and $w_{n}^{+} \rightarrow w^{+} \in X^{+}$. Dividing both sides of $\Phi\left(u_{n}\right) \geq-\mathscr{L}$ by $\left\|u_{n}\right\|^{2}$, we obtain

$$
\begin{aligned}
-\frac{\mathscr{L}}{\left\|u_{n}\right\|^{2}} \leq & \frac{\Phi\left(u_{n}\right)}{\left\|u_{n}\right\|^{2}} \\
= & \frac{1}{2}\left\|w_{n}^{+}\right\|^{2}-\frac{1}{2}\left\|w_{n}^{-}\right\|^{2}-\int_{\mathbb{R}^{N}} \frac{F\left(x, u_{n}\right)}{\left\|u_{n}\right\|^{2}} d x \\
= & \frac{1}{2}\left\|w_{n}^{+}\right\|^{2}-\frac{1}{2}\left\|w_{n}^{-}\right\|^{2} \\
& -\int_{\mathbb{R}^{N}} \frac{F_{1}\left(x, u_{n}\right)}{\left\|u_{n}\right\|^{2}}-\int_{\mathbb{R}^{N}} \frac{F_{2}\left(x, u_{n}\right)}{\left\|u_{n}\right\|^{2}} d x .
\end{aligned}
$$

By (76) and (63), we have

$$
\begin{aligned}
\int_{\mathbb{R}^{N}} \frac{\left|F_{1}\left(x, u_{n}\right)\right|}{\left\|u_{n}\right\|^{2}} d x & \leq \frac{A}{2}\left\|w_{n}\right\|_{L^{2}}^{2} \leq \frac{A}{2 \mu_{0}}\left\|w_{n}\right\|^{2} \\
& =\frac{A}{2 \mu_{0}}\left\|w_{n}^{+}\right\|^{2}+\frac{A}{2 \mu_{0}}\left\|w_{n}^{-}\right\|^{2} .
\end{aligned}
$$

Combining (78) and (79), we obtain

$$
\begin{aligned}
-\frac{\mathscr{L}}{\left\|u_{n}\right\|^{2}} \leq & \left(\frac{1}{2}+\frac{A}{2 \mu_{0}}\right)\left\|w_{n}^{+}\right\|^{2}-\left(\frac{1}{2}-\frac{A}{2 \mu_{0}}\right)\left\|w_{n}^{-}\right\|^{2} \\
& -\int_{\mathbb{R}^{N}} \frac{F_{2}\left(x, u_{n}\right)}{\left\|u_{n}\right\|^{2}} d x .
\end{aligned}
$$

We first consider the case $w \neq 0$. From $\lim _{|t| \rightarrow \infty} f(x, t) /$ $t=+\infty\left(\right.$ see $\left.\left(\mathbf{f}_{2}\right)\right)$, we have

$$
\liminf _{|t| \rightarrow \infty} \frac{F_{2}(x, t)}{t^{2}}=+\infty
$$

Note that, for $x \in\left\{x \in \mathbb{R}^{N} \mid w \neq 0\right\}$, we have $\left|u_{n}(x)\right| \rightarrow+\infty$. Together with (81), this implies that

$$
\liminf _{n \rightarrow \infty} \int_{\left\{x \in \mathbb{R}^{N} \mid w \neq 0\right\}} \frac{F_{2}\left(x, u_{n}\right)}{u_{n}^{2}} w_{n}^{2} d x=+\infty .
$$

By $F_{2} \geq 0$, we obtain

$$
\begin{aligned}
\int_{\mathbb{R}^{N}} \frac{F_{2}\left(x, u_{n}\right)}{\left\|u_{n}\right\|^{2}} d x & =\int_{\mathbb{R}^{N}} \frac{F_{2}\left(x, u_{n}\right)}{u_{n}^{2}} w_{n}^{2} d x \\
& \geq \int_{\left\{x \in \mathbb{R}^{N} \mid w \neq 0\right\}} \frac{F_{2}\left(x, u_{n}\right)}{u_{n}^{2}} w_{n}^{2} d x .
\end{aligned}
$$

Combining (82) and (83) yields

$$
\begin{gathered}
\liminf _{n \rightarrow \infty}\left(\left(\frac{1}{2}+\frac{A}{2 \mu_{0}}\right)\left\|w_{n}^{+}\right\|^{2}-\left(\frac{1}{2}-\frac{A}{2 \mu_{0}}\right)\left\|w_{n}^{-}\right\|^{2}\right. \\
\left.-\int_{\mathbb{R}^{N}} \frac{F_{2}\left(x, u_{n}\right)}{\left\|u_{n}\right\|^{2}} d x\right)=-\infty .
\end{gathered}
$$

This contradicts (80), since $-\mathscr{L} /\left\|u_{n}\right\|^{2} \rightarrow 0$ as $n \rightarrow \infty$.

We then consider the case $w=0$. In this case, $\lim _{n \rightarrow \infty}\left\|w_{n}^{+}\right\|=0$. It follows that

$$
\lim _{n \rightarrow \infty}\left\|w_{n}^{-}\right\|=1
$$

since $\left\|w_{n}\right\|=1$ and $\left\|w_{n}\right\|^{2}=\left\|w_{n}^{+}\right\|^{2}+\left\|w_{n}^{-}\right\|^{2}$. Therefore, the right hand side of $(80)$ is less than $-(1 / 2)\left(1 / 2-A / 2 \mu_{0}\right)$ when $n$ 
is sufficiently large. However, as $n \rightarrow \infty$, the left side of (80) converges to zero. This also induces a contradiction.

Step 3. From (76) and (63), we deduce that, for any $u \in X$,

$$
\begin{aligned}
\Phi(u)= & \frac{1}{2}\left\|u^{+}\right\|^{2}-\frac{1}{2}\left\|u^{-}\right\|^{2}-\int_{\mathbb{R}^{N}} F_{1}(x, u) d x \\
& -\int_{\mathbb{R}^{N}} F_{2}(x, u) d x \\
\leq & \frac{1}{2}\left\|u^{+}\right\|^{2}-\frac{1}{2}\left\|u^{-}\right\|^{2}+\frac{A}{2}\|u\|_{L^{2}}^{2} \\
& -\int_{\mathbb{R}^{N}} F_{2}(x, u) d x \\
\leq & \frac{1}{2}\left\|u^{+}\right\|^{2}-\frac{1}{2}\left\|u^{-}\right\|^{2}+\frac{A}{2 \mu_{0}}\|u\|^{2} \\
& -\int_{\mathbb{R}^{N}} F_{2}(x, u) d x \\
= & \left(\frac{1}{2}+\frac{A}{2 \mu_{0}}\right)\left\|u^{+}\right\|^{2}-\left(\frac{1}{2}-\frac{A}{2 \mu_{0}}\right)\left\|u^{-}\right\|^{2} \\
& -\int_{\mathbb{R}^{N}} F_{2}(x, u) d x .
\end{aligned}
$$

Let

$$
\begin{aligned}
J(u)= & \left(\frac{1}{2}+\frac{A}{2 \mu_{0}}\right)\left\|u^{+}\right\|^{2}-\left(\frac{1}{2}-\frac{A}{2 \mu_{0}}\right)\left\|u^{-}\right\|^{2} \\
& -\int_{\mathbb{R}^{N}} F_{2}(x, u) d x, \quad u \in X .
\end{aligned}
$$

Then by $A<\mu_{0}, F_{2} \geq 0$ and Remark 5 , we deduce that $J$ is $\tau$-upper semicontinuous. Moreover, $J(0)=0<\inf _{N} \Phi$, and, by (86), $\Phi \leq J$ in $X$.

Combining Steps 1-3, we obtain the desired results of this lemma.

\section{Boundedness of $(\bar{C})_{c}$ Sequence and Proof of the Main Results}

According to Definition 6, a sequence $\left\{u_{n}\right\} \subset X$ is called a $(\bar{C})_{c}$ sequence of $\Phi$ if

$$
\begin{array}{r}
\limsup _{n \rightarrow \infty} \Phi\left(u_{n}\right) \leq c, \\
\lim _{n \rightarrow \infty}\left(1+\left\|u_{n}\right\|\right)\left\|\Phi^{\prime}\left(u_{n}\right)\right\|_{X^{\prime}}=0 .
\end{array}
$$

Lemma 11. Suppose that $(\boldsymbol{v})$ and $\left(f_{1}\right)-\left(f_{3}\right)$ are satisfied. Let $\left\{u_{n}\right\}$ be a $(\bar{C})_{c}$ sequence of $\Phi$. Then

$$
\begin{aligned}
& \lim _{n \rightarrow \infty} \int_{\Theta_{n}}\left|u_{n}\right|^{2} d x=0, \\
& \lim _{n \rightarrow \infty} \int_{\Theta_{n}}\left|u_{n}\right|^{p} d x=0,
\end{aligned}
$$

where $\Phi_{n}=\left\{x \in \mathbb{R}^{N}|| u_{n}(x) \mid \geq M\right\}$ and $p$ and $M$ are from $\left(f_{1}\right)$ and $\left(f_{3}\right)$, respectively.
Proof. Let $\widetilde{\omega}_{n}^{+}=\left\{x \in \mathbb{R}^{N} \mid u_{n}(x) \geq M / 2\right\}$ and $v_{n}=\max \left\{u_{n}-\right.$ $M / 2,0\}$. Then

$$
\begin{aligned}
\int_{\mathbb{R}^{N}}\left|\nabla v_{n}\right|^{2} d x & =\int_{\widetilde{\omega}_{n}^{+}}\left|\nabla u_{n}\right|^{2} d x \leq \int_{\mathbb{R}^{N}}\left|\nabla u_{n}\right|^{2} d x, \\
\int_{\mathbb{R}^{N}} v_{n}^{2} d x & =\int_{\widetilde{\omega}_{n}^{+}} v_{n}^{2} d x \leq \int_{\widetilde{\omega}_{n}^{+}} u_{n}^{2} d x \leq \int_{\mathbb{R}^{N}} u_{n}^{2} d x .
\end{aligned}
$$

It follows that $\left\|v_{n}\right\|=O\left(\left\|u_{n}\right\|\right)$. Together with the fact that $\left\{u_{n}\right\}$ is a $(\bar{C})_{c}$ sequence for $\Phi=-J$, this implies that

$$
o(1)=\left\langle\Phi^{\prime}\left(u_{n}\right), v_{n}\right\rangle=-\left\langle J^{\prime}\left(u_{n}\right), v_{n}\right\rangle .
$$

By (6),

$$
a:=\inf _{|t| \geq M / 2, x \in \mathbb{R}^{N}}\left(\frac{f(x, t)}{t}-V_{-}(x)\right)>0 .
$$

Then, by (55) and (91) and the fact that $u_{n} \geq v_{n} \geq 0$ on $\widetilde{\omega}_{n}^{+}$, we obtain

$$
\begin{aligned}
o(1)= & \left\langle J^{\prime}\left(u_{n}\right), v_{n}\right\rangle \\
= & \int_{\mathbb{R}^{N}}\left(\nabla u_{n} \nabla v_{n}+V u_{n} v_{n}\right) d x \\
& +\int_{\mathbb{R}^{N}} f\left(x, u_{n}\right) v_{n} d x \\
= & \int_{\tilde{\omega}_{n}^{+}}\left|\nabla v_{n}\right|^{2} d x+\int_{\widetilde{\omega}_{n}^{+}} V_{+}(x) u_{n} v_{n} d x \\
& +\int_{\widetilde{\omega}_{n}^{+}}\left(\frac{f\left(x, u_{n}\right)}{u_{n}}-V_{-}\right) u_{n} v_{n} d x \\
\geq & \int_{\widetilde{\omega}_{n}^{+}}\left|\nabla v_{n}\right|^{2} d x+a \int_{\widetilde{\omega}_{n}^{+}} v_{n}^{2} d x \\
= & \int_{\mathbb{R}^{N}}\left|\nabla v_{n}\right|^{2} d x+a \int_{\mathbb{R}^{N}} v_{n}^{2} d x,
\end{aligned}
$$

where $V_{+}=V+V_{-} \geq 0$ in $\mathbb{R}^{N}$. Together with the Sobolev inequality $\int_{\mathbb{R}^{N}}\left|\nabla v_{n}\right|^{2} d x+a \int_{\mathbb{R}^{N}} v_{n}^{2} d x \geq C\left(\int_{\mathbb{R}^{N}}\left|v_{n}\right|^{p} d x\right)^{2 / p}$, this yields

$$
\begin{aligned}
& \lim _{n \rightarrow \infty} \int_{\widetilde{\omega}_{n}^{+}}\left|v_{n}\right|^{2} d x=\lim _{n \rightarrow \infty} \int_{\mathbb{R}^{N}}\left|v_{n}\right|^{2} d x=0, \\
& \lim _{n \rightarrow \infty} \int_{\widetilde{\omega}_{n}^{+}}\left|v_{n}\right|^{p} d x=\lim _{n \rightarrow \infty} \int_{\mathbb{R}^{N}}\left|v_{n}\right|^{p} d x=0 .
\end{aligned}
$$

Because

$$
{\omega_{n}^{+}}^{+}:=\left\{x \in \mathbb{R}^{N} \mid u_{n}(x) \geq M\right\} \subset \widetilde{\omega}_{n}^{+}
$$

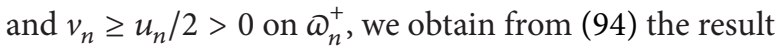

$$
\begin{aligned}
& \lim _{n \rightarrow \infty} \int_{\omega_{n}^{+}}\left|u_{n}\right|^{2} d x=0, \\
& \lim _{n \rightarrow \infty} \int_{\omega_{n}^{+}}\left|u_{n}\right|^{p} d x=0 .
\end{aligned}
$$


Similarly, we can prove that

$$
\begin{aligned}
& \lim _{n \rightarrow \infty} \int_{\omega_{n}^{-}}\left|u_{n}\right|^{2} d x=0, \\
& \lim _{n \rightarrow \infty} \int_{\omega_{n}^{-}}\left|u_{n}\right|^{p} d x=0,
\end{aligned}
$$

where $\omega_{n}^{-}:=\left\{x \in \mathbb{R}^{N} \mid-u_{n}(x) \geq M\right\}$. The result of this lemma follows from (96) and (97).

Lemma 12. Suppose that $(v)$ and $\left(f_{1}\right)-\left(f_{3}\right)$ are satisfied. Let $\left\{u_{n}\right\}$ be $a(\bar{C})_{c}$ sequence of $\Phi$. Then

$$
\sup _{n}\left\|u_{n}\right\|<+\infty
$$

Proof. From $\left(1+\left\|u_{n}\right\|\right)\left\|\Phi^{\prime}\left(u_{n}\right)\right\|_{X^{\prime}} \rightarrow 0$, we get that $\left\langle\Phi^{\prime}\left(u_{n}\right)\right.$, $\left.u_{n}^{ \pm}\right\rangle=o(1)$. Then, by (67), we have

$$
\left\|u_{n}^{ \pm}\right\|^{2}= \pm \int_{\mathbb{R}^{N}} f\left(x, u_{n}\right) u_{n}^{ \pm} d x+o(1)
$$

It follows that

$$
\left\|u_{n}\right\|^{2}=\int_{\mathbb{R}^{N}} f\left(x, u_{n}\right)\left(u_{n}^{+}-u_{n}^{-}\right) d x+o(1) .
$$

From $\left(\mathbf{f}_{1}\right)$ and $\left(\mathbf{f}_{2}\right)$, we deduce that there exists $C_{2}>0$ such that

$$
|f(x, t)| \leq|t|+C_{2}|t|^{p-1}, \quad \forall t \in \mathbb{R} .
$$

Note that $u^{+}$and $u^{-}$are orthogonal with respect to $(\cdot, \cdot)_{L^{2}}$. Then, by (63), we have

$$
\begin{aligned}
\int_{\mathbb{R}^{N}}\left|u^{+}-u^{-}\right|^{2} d x & =\int_{\mathbb{R}^{N}}\left|u^{+}\right|^{2} d x+\int_{\mathbb{R}^{N}}\left|u^{-}\right|^{2} d x \\
& \leq \mu_{0}^{-1}\|u\|^{2}, \quad \forall u \in X .
\end{aligned}
$$

Let

$$
D_{1}=\sup _{\rho \leq|t| \leq M, x \in \mathbb{R}^{N}} \frac{f^{2}(x, t)}{|\widetilde{F}(x, t)|}
$$

and recall that $A=\sup _{|t| \leq \rho, x \in \mathbb{R}^{N}}|f(x, t) / t|$ (see (74)). Using the Hölder inequality, from (100), (101), and (102), we have

$$
\begin{aligned}
\left\|u_{n}\right\|^{2}= & \left(\int_{\left\{x|| u_{n} \mid \leq \rho\right\}}+\int_{\left\{x|\rho<| u_{n} \mid \leq M\right\}}+\int_{\left\{x|| u_{n} \mid>M\right\}}\right) \\
& \cdot f\left(x, u_{n}\right)\left(u_{n}^{+}-u_{n}^{-}\right) d x+o(1) \\
\leq & A\left(\int_{\mathbb{R}^{N}}\left|u_{n}\right|^{2} d x\right)^{1 / 2}\left(\int_{\mathbb{R}^{N}}\left|u_{n}^{+}-u_{n}^{-}\right|^{2} d x\right)^{1 / 2} \\
& +\left(\int_{\left\{x|\rho<| u_{n} \mid \leq M\right\}} f^{2}\left(x, u_{n}\right) d x\right)^{1 / 2} \\
& \cdot\left(\int_{\mathbb{R}^{N}}\left|u_{n}^{+}-u_{n}^{-}\right|^{2} d x\right)^{1 / 2}
\end{aligned}
$$

$$
\begin{aligned}
& +\left(\int_{\left\{x|| u_{n} \mid>M\right\}}\left|u_{n}\right|^{2} d x\right)^{1 / 2} \\
& \cdot\left(\int_{\mathbb{R}^{N}}\left|u_{n}^{+}-u_{n}^{-}\right|^{2} d x\right)^{1 / 2} \\
& +C_{2}\left(\int_{\left\{x|| u_{n} \mid>M\right\}}\left|u_{n}\right|^{p} d x\right)^{(p-1) / p} \\
& +\left(\int_{\mathbb{R}^{N}}\left|u_{n}^{+}-u_{n}^{-}\right|^{p} d x\right)^{1 / p} \\
& \leq A \mu_{0}^{-1}\left\|u_{n}\right\|^{2}+D_{1}^{1 / 2} \mu_{0}^{-1 / 2} \\
& +\left(\int_{\left\{x|\rho<| u_{n} \mid \leq M\right\}}\left|\widetilde{F}\left(x, u_{n}\right)\right| d x\right)^{1 / 2}\left\|u_{n}\right\| \\
& +\mu_{0}^{-1 / 2}\left(\int_{\left\{x|| u_{n} \mid>M\right\}}\left|u_{n}\right|^{2} d x\right)^{1 / 2}\left\|u_{n}\right\| \\
& +C^{\prime} C_{2}\left(\int_{\left\{x|| u_{n} \mid>M\right\}}\left|u_{n}\right|^{p} d x\right)^{(p-1) / p}\left\|u_{n}\right\|
\end{aligned}
$$

where the positive constant $C^{\prime}$ is from the Sobolev inequality $\|u\|_{L^{p}\left(\mathbb{R}^{N}\right)} \leq C^{\prime}\|u\|, \forall u \in X$. By Lemma 11, we have

$$
\int_{\left\{x|| u_{n}(x) \mid \geq M\right\}} u_{n}^{2} d x=o(1),
$$

$$
\int_{\left\{x|| u_{n}(x) \mid \geq M\right\}}\left|u_{n}\right|^{p} d x=o(1) .
$$

Combining (105) with (104) yields

$$
\begin{aligned}
& \left\|u_{n}\right\|^{2} \\
& \leq A \mu_{0}^{-1}\left\|u_{n}\right\|^{2} \\
& \quad+D_{1}^{1 / 2} \mu_{0}^{-1 / 2}\left(\int_{\left\{x|\rho<| u_{n} \mid \leq M\right\}}\left|\widetilde{F}\left(x, u_{n}\right)\right| d x\right)^{1 / 2}\left\|u_{n}\right\| \\
& \quad+o\left(\left\|u_{n}\right\|\right) .
\end{aligned}
$$
obtain

From $\sup _{n} \Phi\left(u_{n}\right) \leq c$ and $\left(1+\left\|u_{n}\right\|\right)\left\|\Phi^{\prime}\left(u_{n}\right)\right\|_{X^{\prime}} \rightarrow 0$, we

$$
\begin{aligned}
o(1)+c & \geq \Phi\left(u_{n}\right)-\frac{1}{2}\left\langle\Phi^{\prime}\left(u_{n}\right), u_{n}\right\rangle \\
& =\int_{\mathbb{R}^{N}} \widetilde{F}\left(x, u_{n}\right) d x .
\end{aligned}
$$


Together with (8), this implies

$$
\begin{aligned}
& \int_{\left\{x|\rho<| u_{n}(x) \mid \leq M\right\}}\left|\widetilde{F}\left(x, u_{n}\right)\right| d x \\
& =\int_{\left\{x|\rho<| u_{n}(x) \mid \leq M\right\}} \widetilde{F}\left(x, u_{n}\right) d x \\
& \leq-\int_{\left\{x|| u_{n}(x) \mid \leq \rho\right\}} \widetilde{F}\left(x, u_{n}\right) d x \\
& \quad-\int_{\left\{x \| u_{n}(x) \mid \geq M\right\}} \widetilde{F}\left(x, u_{n}\right) d x+c+o(1) \\
& \leq \int_{\left\{x|| u_{n}(x) \mid \leq \rho\right\}}\left|\widetilde{F}\left(x, u_{n}\right)\right| d x \\
& \quad+\int_{\left\{x \| u_{n}(x) \mid \geq M\right\}}\left|\widetilde{F}\left(x, u_{n}\right)\right| d x+|c|+o(1) .
\end{aligned}
$$

From $\left(\mathbf{f}_{1}\right)$ and $\left(\mathbf{f}_{2}\right)$, we deduce that there exists $C_{3}>0$ such that

$$
|\widetilde{F}(x, t)| \leq|t|^{2}+C_{3}|t|^{p}, \quad \forall t \in \mathbb{R}
$$

Let $D_{2}=\sup _{|t| \leq \rho, x \in \mathbb{R}^{N}}\left(|\widetilde{F}(x, t)| / t^{2}\right)$. Combining (109), (105) with (108) yields

$$
\begin{aligned}
& \int_{\left\{x|\rho<| u_{n}(x) \mid \leq M\right\}}\left|\widetilde{F}\left(x, u_{n}\right)\right| d x \\
& \leq \int_{\left\{x|| u_{n}(x) \mid \leq \rho\right\}}\left|\widetilde{F}\left(x, u_{n}\right)\right| d x \\
& \quad+\int_{\left\{x|| u_{n}(x) \mid \geq M\right\}}\left|\widetilde{F}\left(x, u_{n}\right)\right| d x+|c|+o(1) \\
& \leq D_{2} \int_{\left\{x|| u_{n}(x) \mid \leq \rho\right\}} u_{n}^{2} d x+\int_{\left\{x|| u_{n}(x) \mid \geq M\right\}} u_{n}^{2} d x \\
& \quad+C_{3} \int_{\left\{x|| u_{n}(x) \mid \geq M\right\}}\left|u_{n}\right|^{p} d x+|c|+o(1) \\
& \leq D_{2} \mu_{0}^{-1}\left\|u_{n}\right\|^{2}+\int_{\left\{x|| u_{n}(x) \mid \geq M\right\}} u_{n}^{2} d x \\
& \quad+C_{3} \int_{\left\{x|| u_{n}(x) \mid \geq M\right\}}\left|u_{n}\right|^{p} d x+|c|+o(1) \\
& =D_{2} \mu_{0}^{-1}\left\|u_{n}\right\|^{2}+|c|+o(1) .
\end{aligned}
$$

Together with (106), this implies

$$
\left\|u_{n}\right\|^{2} \leq\left(A \mu_{0}^{-1}+D_{1}^{1 / 2} D_{2}^{1 / 2} \mu_{0}^{-1}\right)\left\|u_{n}\right\|^{2}+O\left(\left\|u_{n}\right\|\right) .
$$

From (9), we have

$$
A \mu_{0}^{-1}+D_{1}^{1 / 2} D_{2}^{1 / 2} \mu_{0}^{-1}<1
$$

The boundedness of $\left\{\left\|u_{n}\right\|\right\}$ immediately follows from (111) and (112).
Proof of Theorem 1. From the proof of Lemma 6.15 in [23], we know that $\Phi^{\prime}$ is weakly sequentially continuous. Moreover, it is easy to see that $\sup _{M} \Phi<+\infty$. Then by Lemmas 10 and 12 and Corollary 8 , we deduce that there exists a bounded $(\bar{C})_{c}$ sequence $\left\{u_{n}\right\}$ for $\Phi$ with $c=\sup _{M} \Phi$ and inf ${ }_{n}\left\|\left|u_{n} \|\right|>0\right.$. Up to a subsequence, either

(i) $\lim _{n \rightarrow \infty} \sup _{y \in \mathbb{R}^{N}} \int_{B_{1}(y)}\left|u_{n}\right|^{2} d x=0$, or

(ii) there exist $\varrho>0$ and $a_{n} \in \mathbb{Z}^{N}$ such that $\int_{B_{1}\left(a_{n}\right)}\left|u_{n}\right|^{2}$ $d x \geq \varrho$.

If (i) occurs, using the Lions lemma (see, e.g., [23, Lemma 1.21]), a similar argument as for the proof of [20, Lemma 3.6] shows that

$$
\lim _{n \rightarrow \infty} \int_{\mathbb{R}^{N}} f\left(x, u_{n}\right) u_{n}^{ \pm} d x=0 .
$$

Then by (100), we have $\left\|u_{n}\right\| \rightarrow 0$. This contradicts $\inf _{n}\left|\left\|u_{n}|\||>0\right.\right.$. Therefore, case (i) cannot occur. As case (ii) therefore occurs, $w_{n}=u_{n}\left(\cdot+a_{n}\right)$ satisfies $w_{n} \rightarrow u_{0} \neq 0$. From $\left(1+\left\|w_{n}\right\|\right)\left\|\Phi^{\prime}\left(w_{n}\right)\right\|_{X^{\prime}}=\left(1+\left\|u_{n}\right\|\right)\left\|\Phi^{\prime}\left(u_{n}\right)\right\|_{X^{\prime}} \rightarrow 0$ and the weakly sequential continuity of $\Phi^{\prime}$, we have that $\Phi^{\prime}\left(u_{0}\right)=0$. Therefore, $u_{0}$ is a nontrivial solution of (1). This completes the proof.

Proof of Corollary 2. Let

$$
f_{\lambda}(t)=|t|^{q-2} t-\lambda|t|^{p-2} t, \quad t \in \mathbb{R} .
$$

Since $2<p<q<2^{*}$, we deduce that $f_{\lambda}$ satisfies $\left(\mathbf{f}_{\mathbf{1}}\right)$ and $\left(\mathbf{f}_{\mathbf{2}}\right)$. Because $q>p$, there exists $M>0$ such that

$$
\inf _{|t| \geq M / 2}\left(|t|^{q-2}-|t|^{p-2}\right)>\max _{\mathbb{R}^{N}} V_{-} .
$$

It follows that, for $0<\lambda \leq 1, f_{\lambda}$ satisfies (6).

Let

$$
\rho=\left(\frac{q \lambda}{p}\right)^{1 /(q-p)}
$$

Then, for $|t| \geq \rho$,

$$
F_{\lambda}(t)=\int_{0}^{t} f_{\lambda}(s) d s=\frac{1}{q}|t|^{q}-\frac{\lambda}{p}|t|^{p} \geq 0 ;
$$

that is, $F_{\lambda}$ satisfies (7).

Let

$$
\begin{aligned}
\widetilde{F}_{\lambda}(t) & =\frac{1}{2} t f_{\lambda}(t)-F_{\lambda}(t) \\
& =\left(\frac{1}{2}-\frac{1}{q}\right)|t|^{q}-\lambda\left(\frac{1}{2}-\frac{1}{p}\right)|t|^{p} .
\end{aligned}
$$

If $|t| \geq \rho$, then

$$
\begin{aligned}
\widetilde{F}_{\lambda}(t) & =\left(\frac{1}{2}-\frac{1}{q}\right)|t|^{q}-\lambda\left(\frac{1}{2}-\frac{1}{p}\right)|t|^{p} \\
& \geq \frac{q-p}{2 q}|t|^{q} .
\end{aligned}
$$

This shows that $\widetilde{F}_{\lambda}$ satisfies (8). 
It follows from

$$
0 \leq|t|^{q-2}-\lambda|t|^{p-2} \leq|t|^{q-2} \quad \text { if }|t| \geq \rho
$$

that

$$
f_{\lambda}^{2}(t)=\left(|t|^{q-2}-\lambda|t|^{p-2}\right)^{2} t^{2} \leq|t|^{2 q-2} \quad \text { if }|t| \geq \rho .
$$

Let $\lambda$ be sufficiently small such that $M>\rho$. Combining (119) with (121) yields

$$
\frac{f_{\lambda}^{2}(t)}{\widetilde{F}_{\lambda}(t)} \leq \frac{2 q}{q-p}|t|^{q-2} \leq \frac{2 q}{q-p} M^{q-2} \quad \text { if } \rho \leq|t| \leq M .
$$

Moreover, if $|t| \leq \rho$, we have

$$
\begin{aligned}
\left|\frac{f_{\lambda}(t)}{t}\right| & =\left.|| t\right|^{q-2}-\lambda|t|^{p-2} \mid \leq \rho^{q-2}+\lambda \rho^{p-2}, \\
\left|\frac{\widetilde{F}_{\lambda}(t)}{t^{2}}\right| & \leq\left(\frac{1}{2}-\frac{1}{q}\right)|t|^{q-2}+\lambda\left(\frac{1}{2}-\frac{1}{p}\right)|t|^{p-2} \\
& \leq\left(\frac{1}{2}-\frac{1}{q}\right) \rho^{q-2}+\lambda\left(\frac{1}{2}-\frac{1}{p}\right) \rho^{p-2} .
\end{aligned}
$$

Let $\lambda>0$ be sufficiently small such that

$$
\begin{aligned}
\rho^{q-2}+ & \lambda \rho^{p-2}+\left(\frac{2 q}{q-p} M^{q-2}\right)^{1 / 2} \\
& \cdot\left(\left(\frac{1}{2}-\frac{1}{q}\right) \rho^{q-2}+\lambda\left(\frac{1}{2}-\frac{1}{p}\right) \rho^{p-2}\right)^{1 / 2}<\mu_{0} .
\end{aligned}
$$

It follows from (122)-(124) that $f_{\lambda}$ and $\widetilde{F}_{\lambda}$ satisfy (9). Therefore, we verified that $f_{\lambda}, F_{\lambda}$, and $\widetilde{F}_{\lambda}$ satisfy (6)-(9) if $\lambda>0$ is sufficiently small. The result of this corollary immediately follows from Theorem 1.

\section{Conflict of Interests}

The authors declare that there is no conflict of interests regarding the publication of this paper.

\section{Acknowledgments}

The authors would like to thank the anonymous referees for their comments and suggestions on the paper. Shaowei Chen was supported by Science Foundation of Huaqiao University and Promotion Program for Young and Middle-aged Teacher in Science and Technology Research of Huaqiao University (ZQN-PY119).

\section{References}

[1] M. Reed and B. Simon, Methods of Modern Mathematical Physics, IV: Analysis of Operators, Academic Press, New York, NY, USA, 1978.

[2] S. Alama and Y. Y. Li, "On "multibump" bound states for certain semilinear elliptic equations," Indiana University Mathematics Journal, vol. 41, no. 4, pp. 983-1026, 1992.
[3] C. Troestler and M. Willem, "Nontrivial solution of a semilinear Schrödinger equation," Communications in Partial Differential Equations, vol. 21, no. 9-10, pp. 1431-1449, 1996.

[4] W. Kryszewski and A. Szulkin, "Generalized linking theorem with an application to a semilinear Schrödinger equation," Advances in Differential Equations, vol. 3, no. 3, pp. 441-472, 1998.

[5] A. A. Pankov and K. Pflüger, "On a semilinear Schrödinger equation with periodic potential," Nonlinear Analysis Series A: Theory, Methods \& Applications, vol. 33, no. 6, pp. 593-609, 1998.

[6] A. Pankov, "Periodic nonlinear Schrödinger equation with application to photonic crystals," Milan Journal of Mathematics, vol. 73, no. 1, pp. 259-287, 2005.

[7] S. Chen and D. Zhang, "Existence of nontrivial solutions for asymptotically linear periodic Schrödinger equations," Complex Variables and Elliptic Equations, 2014.

[8] Y. Ding, Variational Methods for Strongly Indefinite Problems. Interdisciplinary Mathematical Sciences, vol. 7, World Scientific, Hackensack, NJ, USA, 2007.

[9] Y. Ding and C. Lee, "Multiple solutions of Schrödinger equations with indefinite linear part and super or asymptotically linear terms," Journal of Differential Equations, vol. 222, no. 1, pp. 137-163, 2006.

[10] G. Li and A. Szulkin, "An asymptotically periodic Schrödinger equation with indefinite linear part," Communications in Contemporary Mathematics, vol. 4, no. 4, pp. 763-776, 2002.

[11] N. Ackermann, "A nonlinear superposition principle and multibump solutions of periodic Schrödinger equations," Journal of Functional Analysis, vol. 234, no. 2, pp. 277-320, 2006.

[12] C. J. Batkam and F. Colin, "Generalized fountain theorem and applications to strongly indefinite semilinear problems," Journal of Mathematical Analysis and Applications, vol. 405, no. 2, pp. 438-452, 2013.

[13] S. Chen, "Multi-bump solutions for a strongly indefinite semilinear Schrödinger equation without symmetry or convexity assumptions," Nonlinear Analysis: Theory, Methods \& Applications, vol. 68, no. 10, pp. 3067-3102, 2008.

[14] W. Kryszewski and A. Szulkin, "Infinite-dimensional homology and multibump solutions," Journal of Fixed Point Theory and Applications, vol. 5, no. 1, pp. 1-35, 2009.

[15] S. Liu, "On superlinear Schrödinger equations with periodic potential," Calculus of Variations and Partial Differential Equations, vol. 45, no. 1-2, pp. 1-9, 2012.

[16] A. Szulkin and T. Weth, "Ground state solutions for some indefinite variational problems," Journal of Functional Analysis, vol. 257, no. 12, pp. 3802-3822, 2009.

[17] M. Yang, "Ground state solutions for a periodic Schrödinger equation with superlinear nonlinearities," Nonlinear Analysis. Theory, Methods \& Applications, vol. 72, no. 5, pp. 2620-2627, 2010.

[18] M. Willem and W. Zou, "On a Schrödinger equation with periodic potential and spectrum point zero," Indiana University Mathematics Journal, vol. 52, no. 1, pp. 109-132, 2003.

[19] M. Yang, W. Chen, and Y. H. Ding, "Solutions for periodic Schrödinger equation with spectrum zero and general superlinear nonlinearities," Journal of Mathematical Analysis and Applications, vol. 364, no. 2, pp. 404-413, 2010.

[20] A. Szulkin and W. Zou, "Homoclinic orbits for asymptotically linear Hamiltonian systems," Journal of Functional Analysis, vol. 187, no. 1, pp. 25-41, 2001. 
[21] A. Pankov, "Gap solitons in periodic discrete nonlinear Schrödinger equations with saturable nonlinearities," Journal of Mathematical Analysis and Applications, vol. 371, no. 1, pp. 254265, 2010.

[22] S. Chen and D. Zhang, "Existence of nontrivial solutions for periodic Schrödinger equations with new nonlinearities," Abstract and Applied Analysis, vol. 2014, Article ID 539639, 10 pages, 2014.

[23] M. Willem, Minimax Theorems, Progress in Nonlinear Differential Equations and their Applications, 24, Birkhäuser Boston, Boston, MA, USA, 1996.

[24] S. Chen and C. Wang, "An infinite-dimensional linking theorem without upper semi-continuous assumption and its applications," Journal of Mathematical Analysis and Applications, vol. 420, no. 2, pp. 1552-1567, 2014.

[25] G. E. Ladas and V. Lakshmikantham, Differential Equations in Abstract Spaces, vol. 85 of Mathematics in Science and Engineering, Academic Press, New York, NY, USA, 1972. 


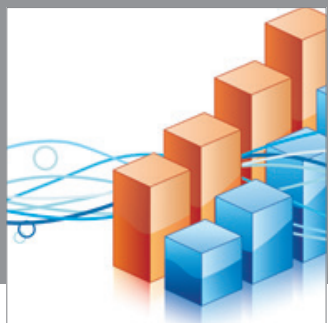

Advances in

Operations Research

mansans

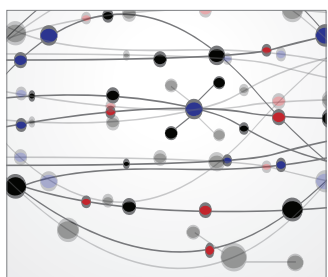

The Scientific World Journal
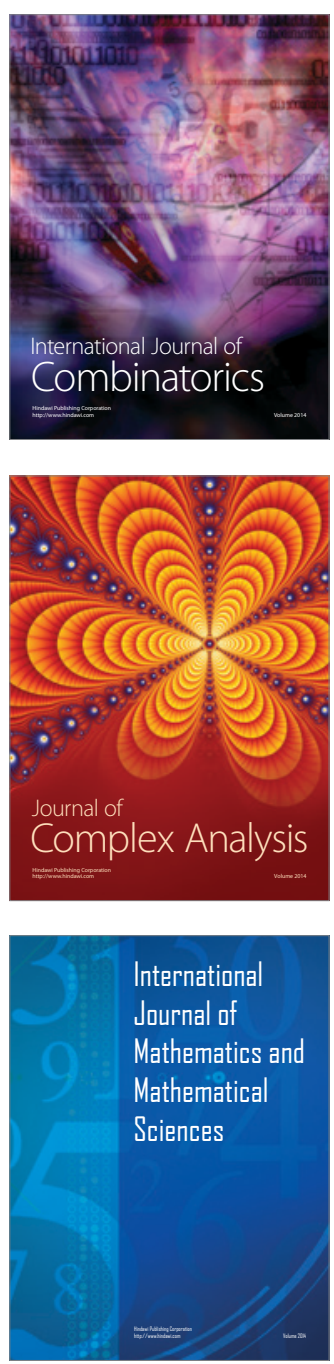
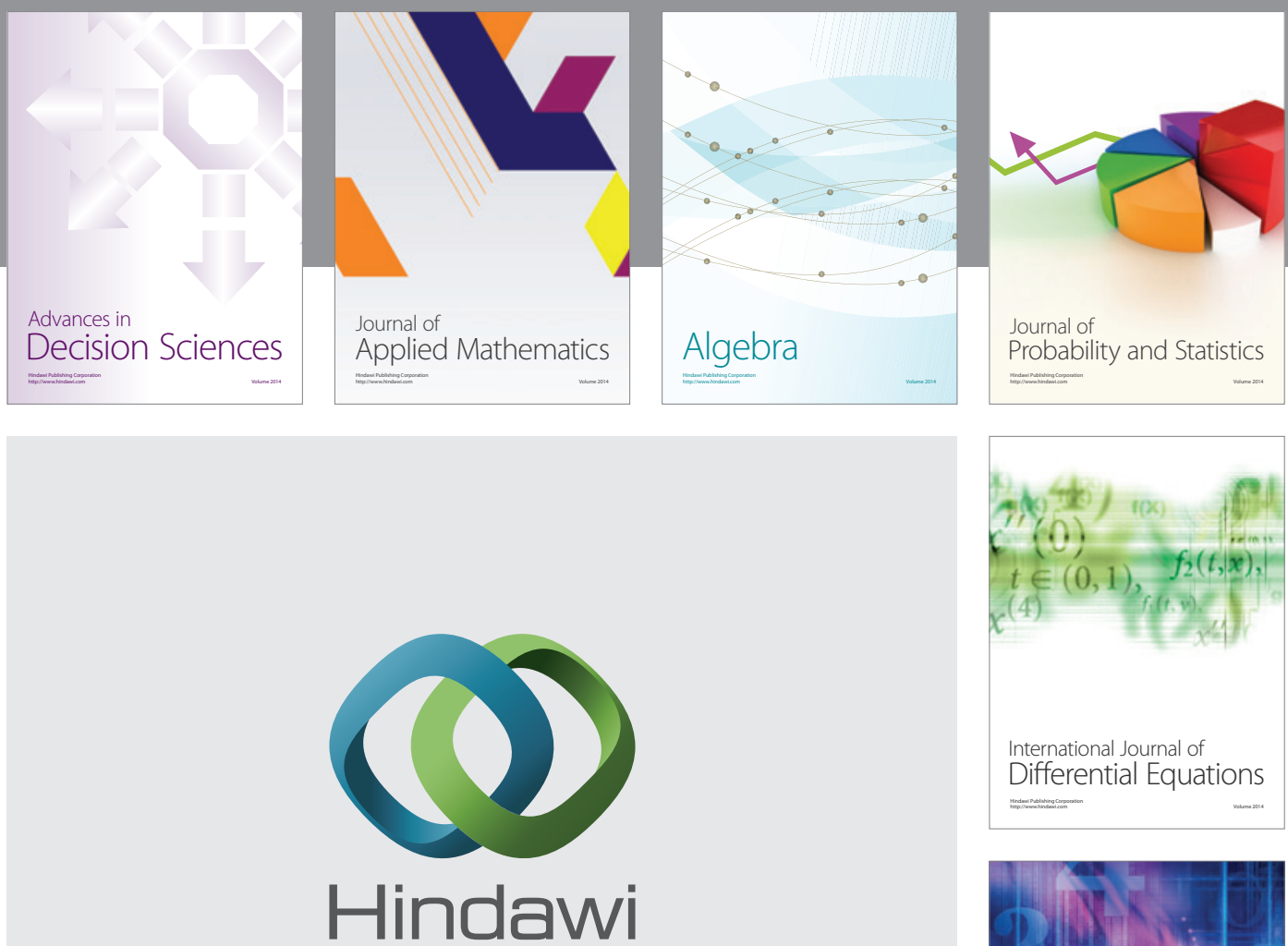

Submit your manuscripts at http://www.hindawi.com
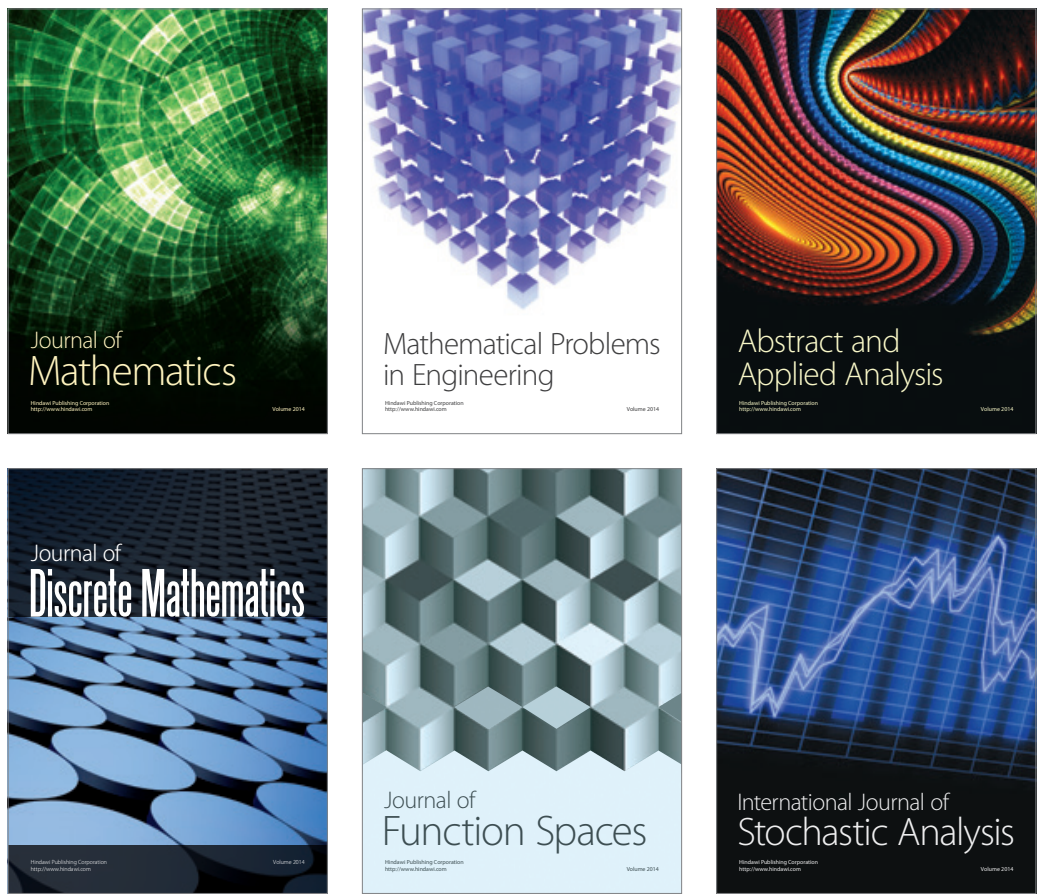

Journal of

Function Spaces

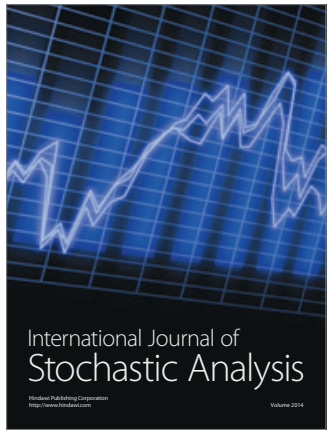

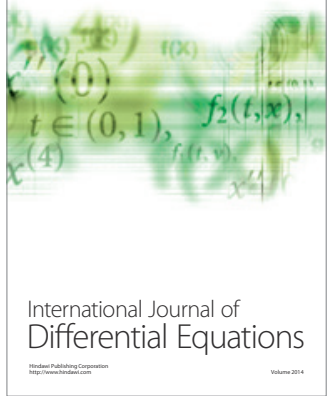
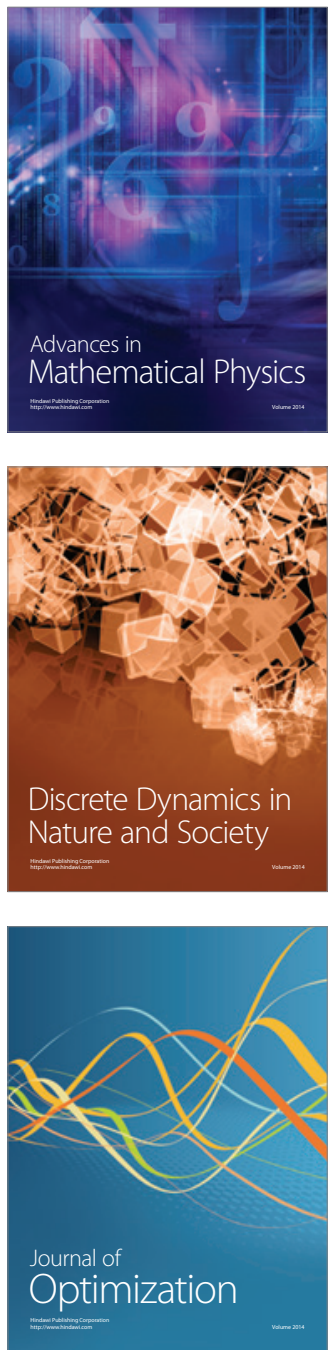